\title{
Demographic, socioeconomic, and health correlates of unmet need for mental health treatment in the United States, 2002-16: evidence from the national surveys on drug use and health
}

Justin C. Yang ${ }^{1 *+} \mathbb{D}$, Andres Roman-Urrestarazu ${ }^{1+}$, Martin McKee $^{2}$ and Carol Brayne ${ }^{1}$

\begin{abstract}
Background: Unmet need for mental health services remains high in the United States and is disproportionately concentrated in some groups. The scale and nature of these disparities have not been fully elucidated and bear further scrutiny. As such, in this study, we examine the demographic, socioeconomic, and health correlates of unmet need for mental health treatment as well as the reasons for unmet need.

Methods: We draw upon the National Survey for Drug Use and Health (NSDUH) from 2002 to 16 for adults aged 18 and over in the United States $(n=579,017)$. Using multivariable logistic regression, we simultaneously model the demographic, socioeconomic, and health correlates of unmet need for mental health treatment from 2002 to 16 . We also analyse the reasons for unmet need expressed by these populations, reasons which include cost, perceived stigma, minimisation of symptoms, low perceived effectiveness of treatment, and structural barriers.

Results: Major characteristics associated with increased odds of unmet need include past year substance abuse or dependence (other than hallucinogens and sedatives), fair, poor, or very poor health, being female, and an educational attainment of college or higher. With respect to reasons for unmet need, cost was most often cited, followed by perceived stigma, structural barriers, and minimisation. Characteristics associated with increased odds of indicating cost as a reason for unmet need include: being uninsured or aged 26-35. Minimisation and low perceived effectiveness are mentioned by high-income persons as reasons for unmet need. College-educated persons and women had higher odds of citing structural barriers as a reason for unmet need.

Conclusions: The correlates and causes of unmet need highlight the intersectionality of individual health needs with implications on addressing inequities in mental health policy and practice.
\end{abstract}

Keywords: Mental health treatment, Unmet need

\footnotetext{
* Correspondence: jcy25@medschl.cam.ac.uk

†Justin C. Yang and Andres Roman-Urrestarazu contributed equally to this manuscript as co-first authors.

'Department of Public Health \& Primary Care, Institute of Public Health,

University of Cambridge, Forvie Site, Robinson Way, Cambridge CB2 OSR, UK

Full list of author information is available at the end of the article
}

(c) The Author(s). 2019 Open Access This article is distributed under the terms of the Creative Commons Attribution 4.0 International License (http://creativecommons.org/licenses/by/4.0/), which permits unrestricted use, distribution, and reproduction in any medium, provided you give appropriate credit to the original author(s) and the source, provide a link to the Creative Commons license, and indicate if changes were made. The Creative Commons Public Domain Dedication waiver (http://creativecommons.org/publicdomain/zero/1.0/) applies to the data made available in this article, unless otherwise stated. 


\section{Introduction}

Mental disorders and substance use disorders are major contributors to years lived with disability in the United States $(3,536,895.4$ years lived with disability [YLDs] in 2016, or 1095.45 YLDs per 100,000 population) [1]. They are common, with data from the National Survey on Drug Use and Health (2010-2012) finding that $18.4 \%$ of adults had a mental illness and $8.6 \%$ reported substance abuse/dependence, while $2.2 \%$ had both [2]. Federal policymakers have responded with a variety of legislative approaches, notably the Mental Health Parity and Addiction Equity Act of 2008 (MHPAEA), while the Patient Protection and Affordable Care Act of 2010 (ACA) includes specific provisions for mental health [3]. Yet, despite these policies, there are large and persisting disparities in access to and receipt of mental health services. Individual studies have identified a range of underserved populations, which include certain ethnic minorities [4] and those lacking insurance [5]. Moreover, difficulties in accessing adequate mental health treatment have been documented among individuals with substance use disorders [6]. These previous studies signal an urgent need to address disparities in mental health treatment.

However, describing those whose needs for care are unmet is only a first step. It is also necessary to understand the reasons, if an appropriate policy response is to be developed. Possible explanations may lie within the affected individual or within the health care system.

In this study, we seek to characterise the demographic, socioeconomic, and health correlates of unmet need for mental health care among Americans, using data from the period 2002 to 2016 and to understand the reasons their needs are not being met. To our knowledge, this is the first attempt to simultaneously analyse these correlates at once. With respect to the reasons for unmet need, we draw upon and extend existing analyses which have reviewed these causes which include: cost, perceived stigma, minimisation of symptoms, low perceived effectiveness of treatment, and structural barriers [5].

\section{Methods}

This study utilises data from the 2002-16 waves of the NSDUH, a nationally representative, annual household survey of civilian, non-institutionalised individuals aged 12 and above in the United States [7-21].The NSDUH collects data on measures of mental health, including substance use and unmet need for mental health treatment, as well as a range of demographic, socioeconomic, and health-related variables [7-21]. Households are selected from all 50 states and the District Columbia, excluding individuals with no fixed address, active duty military personnel, and individuals living in institutionalised facilities [7-21]. Sampling and analytical weights for the NSDUH datasets were provided by the Substance
Abuse and Mental Health Services Administration (SAMHSA) to address unit- and individual-level nonresponse [7-21]. In the current study, the only exclusion criterion we applied to the dataset was to exclude respondents aged 17 or below given our interest in the adult population exclusively.

Age at time of survey was categorised as: 18-25, 26$34,35-49$, and 50 or over. Sex was coded as either male or female. Ethnicity was coded as non-Hispanic White, non-Hispanic Black, non-Hispanic Native American or Alaskan Native, non-Hispanic Native Hawaiian or Pacific Islander, non-Hispanic Asian, non-Hispanic mixed, or Hispanic. Marital status was coded as never married, married, widowed, divorced, or separated. Population density was coded as metropolitan statistical area (MSA) or core based statistical area (CBSA) 1 million or more ("urban"), MSA or CBSA with fewer than 1 million ("suburban"), or non-MSA/CBSA ("rural") based on data from the 2000 or 2010 census and information from the Core Based Statistical Area classifications compiled by the Office of Management and Budget (OMB) [7-21].

Education was coded as the highest level reached from among elementary school, middle school, high school or college and higher. Employment was coded as full-time employed, part-time employed, unemployed, or other (defined as those not in the labour force such as students, retirees, or disabled individuals). Annual household income was coded as less than $\$ 20,000$, between $\$ 20,000$ and $\$ 49,999$, between $\$ 50,000$ and $\$ 74,999$, and $\$ 75,000$ or greater. A dichotomous variable was created to indicate whether the respondent was a recipient of a government assistance program (i.e. Supplemental Security Income [SSI], food stamps, cash assistance, and/or non-cash assistance). Insurance provider was coded as privately insured, insured by Medicare, insured by Medicaid, insured by Tricare or Veterans Administration (VA), uninsured, or other.

Self-rated health was dichotomised into two categories: those reporting excellent, very good, or good self-rated health and those reporting fair, poor, or very poor selfrated health. Dichotomous variables indicating past-year substance abuse or dependence were coded for each of: alcohol, pain relievers, cocaine, hallucinogens, heroin, inhalants, marijuana, sedatives, stimulants, and tranquilizers.

Expressed unmet need for mental health treatment ("unmet need") was defined as meeting both the following conditions: (a) perceiving of a need for mental health treatment in the past year and (b) perceiving receipt of insufficient mental health treatment in the past year, including those adults who had received mental health treatment in the past year but perceived a further need that was unmet [7-21]. A dichotomous variable was created to code for responses to the question, "During the past 12 months, was there any time when you needed 
mental health treatment or counselling for yourself but didn't get it?" [7-21]. Among individuals expressing unmet need for mental health treatment, further dichotomous variables were coded to indicate whether the unmet need was due to one or more of the following reasons: cost, stigma, minimisation, low perceived treatment effectiveness, structural barriers or other reasons using definitions set by SAMHSA [7-21] and from established literature [5]. The specification of these variables are shown in Table 1.

Item non-response was addressed using imputation based on predictive mean neighbourhood (PMN), as has been done with NSDUH datasets since 1999 [7-21]. Imputation is performed most for variables pertaining to ethnicity and government assistance, drawing upon responses to other related questions where logical errors and item non-response exist, and to a lesser degree for education, marital status, income, and health insurer [721]. The percentage of observations with imputed values ranges from $<0.1$ to $4 \%$ based on variable [7-21].

Statistical analyses were performed in Stata 14. We conducted bivariate descriptive analysis of our primary dichotomous variable of interest, unmet need for mental health treatment, and multiple maximum-likelihood logit regression with weighted least squares on social, economic, and

Table 1 Reasons for unmet need and corresponding survey responses

\begin{tabular}{|c|c|}
\hline $\begin{array}{l}\text { Reasons for Unmet } \\
\text { Need }\end{array}$ & Survey Responses \\
\hline \multirow[t]{3}{*}{ Cost } & Could not afford the costs of treatment \\
\hline & $\begin{array}{l}\text { Health insurance does not cover mental } \\
\text { health treatment }\end{array}$ \\
\hline & $\begin{array}{l}\text { Health insurance does not cover enough } \\
\text { for mental health treatment }\end{array}$ \\
\hline \multirow[t]{5}{*}{ Stigma } & $\begin{array}{l}\text { Might cause neighbours to have a negative } \\
\text { opinion }\end{array}$ \\
\hline & Might have a negative effect on job \\
\hline & Did not want others to find out \\
\hline & Concerned about confidentiality \\
\hline & Concerned about being committed/medicated \\
\hline \multirow[t]{2}{*}{ Minimisation } & $\begin{array}{l}\text { Did not feel that they needed mental health } \\
\text { treatment }\end{array}$ \\
\hline & $\begin{array}{l}\text { Felt that they could handle the problem without } \\
\text { treatment }\end{array}$ \\
\hline $\begin{array}{l}\text { Low Perceived } \\
\text { Effectiveness of } \\
\text { Treatment }\end{array}$ & $\begin{array}{l}\text { Did not feel that mental health treatment would } \\
\text { help }\end{array}$ \\
\hline \multirow[t]{3}{*}{ Structural Barriers } & $\begin{array}{l}\text { Did not know where to go for mental health } \\
\text { treatment }\end{array}$ \\
\hline & Did not have the time \\
\hline & Inconvenient to attend mental health treatment \\
\hline Other Reasons & Other reason than those listed above \\
\hline
\end{tabular}

health correlates, with and without adjustment, to assess the demographic, socioeconomic, and health correlates of unmet mental health treatment both individually and simultaneously adjusting for all other correlates. Further analyses of our secondary dichotomous variables of interest indicating reason(s) for unmet need were also conducted using multiple maximum-likelihood logit regressions with weighted least squares on social, economic, and health correlates. Given the NSDUH's complex sampling design, all analyses were weighted using analytical weights provided by SAMHSA with each annual dataset.

\section{Results}

Descriptive characteristics of our sample are shown in Table 2. From 2002 to 16, between 36,000-43,000 adults aged 18 or above were surveyed annually, yielding a total study population of 579,017 . Table 3 shows the reasons respondents gave for past-year unmet need for mental health treatment from 2002 to 16.

\section{Unmet need}

Unadjusted and adjusted odds of reporting unmet need among those perceiving a need for mental health treatment in the past year according to a range of demographic, socioeconomic, and health characteristics are shown in Table 4. Factors increasing the odds of reported unmet need included: being female, attaining an educational level of college or higher, receiving government assistance, reporting fair, poor, or very poor health, and being insured by Medicaid, Tricare or VA, or not having health insurance. In addition, with the exception of hallucinogens and sedatives, past-year abuse or dependence on any substance increased the odds of unmet need. On the other hand, attributes of decreased odds of unmet need included: age over 34, being married or widowed, and having a household income of $\$ 50,000$ or more.

\section{Reasons for unmet need}

Table 5 shows the results of adjusted logistic regression of individual demographic, socioeconomic, and health characteristics associated reported reasons for unmet need among respondents indicating past-year unmet need for mental health treatment.

Cost was more likely to be cited as a reason for unmet need by subjects between 26 and 49 years of age, those reporting fair, poor, or very poor self-rated health, and those with who reported being uninsured. Those living in suburban or rural areas were more likely to indicate perceived stigma and minimisation as reasons for unmet need. Respondents with fair, poor, or very poor self-rated health or who reported past year abuse or dependence on either alcohol or pain relievers were also more likely to cite perceived stigma as a reason for unmet need. Respondents with an annual household income of $\$ 50$, 
Table 2 Sample characteristics for adults age 18 and over by percentage (\%), 2002-16

\begin{tabular}{|c|c|c|c|c|c|c|c|c|c|c|c|c|c|c|c|}
\hline Year & 2002 & 2003 & 2004 & 2005 & 2006 & 2007 & 2008 & 2009 & 2010 & 2011 & 2012 & 2013 & 2014 & 2015 & 2016 \\
\hline$n$ & 36370 & 37026 & 37308 & 37227 & 36965 & 37708 & 37504 & 37707 & 38919 & 39133 & 37869 & 37424 & 41671 & 43561 & 42625 \\
\hline
\end{tabular}

18-25

26-34

35-49

$\geq 50$

Female

Ethnicity

Non-Hispanic White

Non-Hispanic Black

Non-Hispanic Native American or Alaskan Native

Non-Hispanic Native Hawaiian or Pacific Islander

Non-Hispanic Asian

Non-Hispanic Mixed

Hispanic

Marital Status

Never married

Married

Widowed

Divorced or Separated

MSA/CBSA Size

1 million or more

Fewer than 1 million

Not a MSA/CBSA

Educational Attainment

Elementary School

Middle School

High School

College or Higher

Employment Status

Full Time

Part Time

Unemployed

Other (including not in labour force)

Annual Household Income

Less than $\$ 20,000$

$\$ 20,000-\$ 49,999$

$\$ 50,000-\$ 74,999$

$\$ 75,000$ or more

Receives Government Assistance Self-Rated Health

Good, very good, or excellent

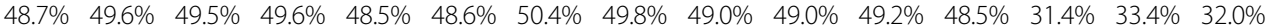

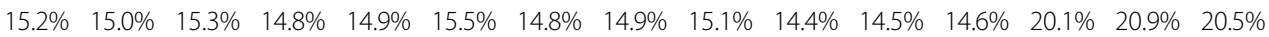

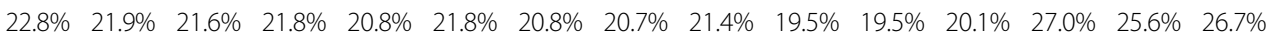

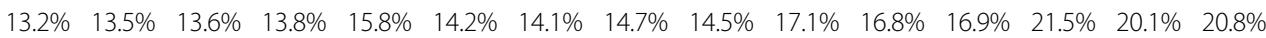

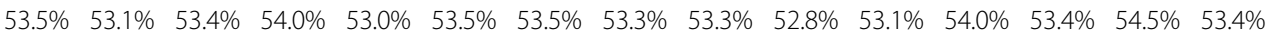

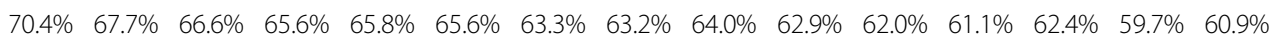

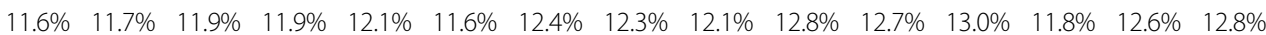

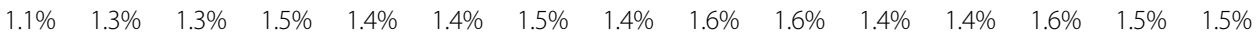

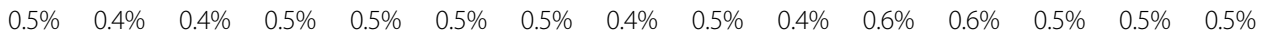

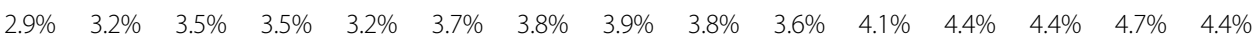

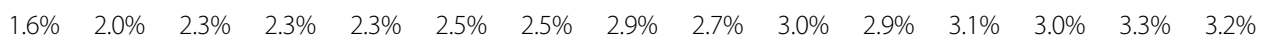

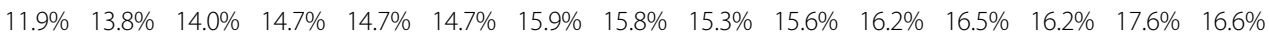

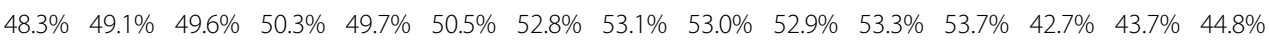

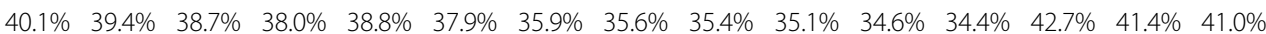
$2.5 \% \quad 2.3 \% \quad 2.4 \% \quad 2.4 \% \quad 2.8 \% \quad 2.4 \% \quad 2.3 \% \quad 2.3 \% \quad 2.5 \% \quad 2.5 \% \quad 2.6 \% \quad 2.5 \% \quad 3.1 \% \quad 3.7 \% \quad 3.0 \%$

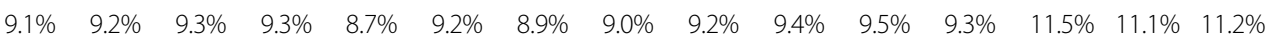

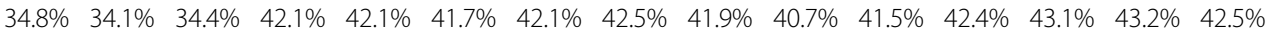

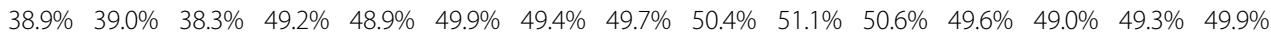

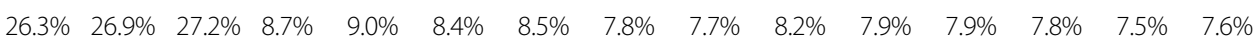

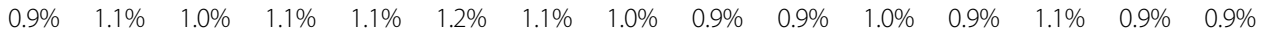
$2.8 \% \quad 3.0 \% \quad 2.9 \% \quad 3.2 \% \quad 3.0 \% \quad 2.9 \% \quad 2.6 \% \quad 2.6 \% \quad 2.6 \% \quad 2.4 \% \quad 2.2 \% \quad 2.3 \% \quad 2.4 \% \quad 2.5 \% \quad 2.0 \%$

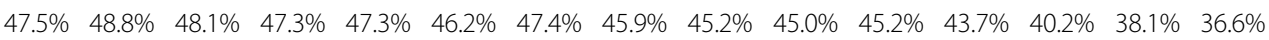

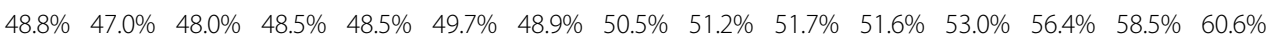

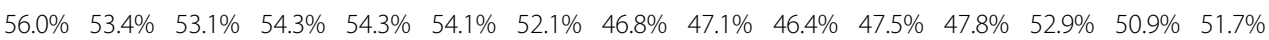

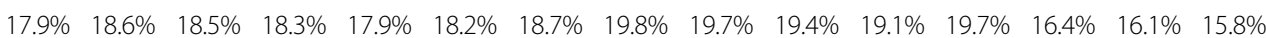

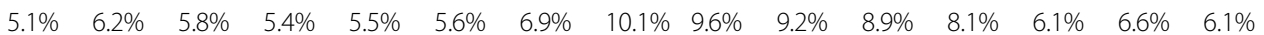

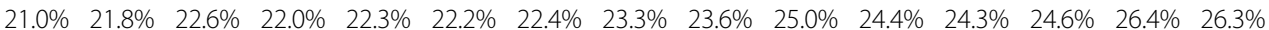

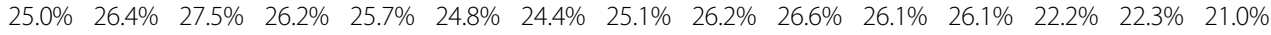

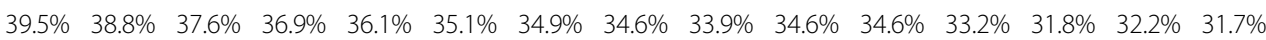

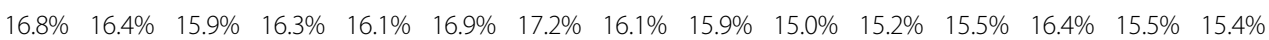

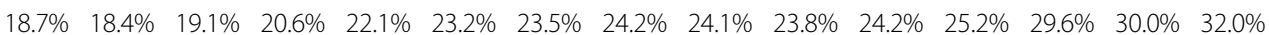

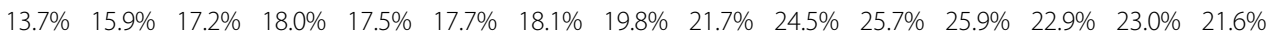

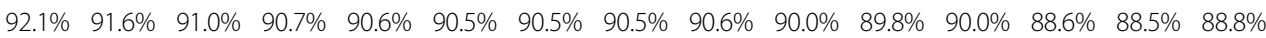


Table 2 Sample characteristics for adults age 18 and over by percentage (\%), 2002-16 (Continued)

\begin{tabular}{|c|c|c|c|c|c|c|c|c|c|c|c|c|c|c|c|}
\hline Year & 2002 & 2003 & 2004 & 2005 & 2006 & 2007 & 2008 & 2009 & 2010 & 2011 & 2012 & 2013 & 2014 & 2015 & 2016 \\
\hline$n$ & 36370 & 37026 & 37308 & 37227 & 36965 & 37708 & 37504 & 37707 & 38919 & 39133 & 37869 & 37424 & 41671 & 43561 & 42625 \\
\hline Fair, poor, or very poor & $7.9 \%$ & $8.4 \%$ & $9.0 \%$ & $9.3 \%$ & $9.4 \%$ & $9.5 \%$ & $9.5 \%$ & $9.5 \%$ & $9.4 \%$ & $10.0 \%$ & $10.2 \%$ & $10.0 \%$ & $11.4 \%$ & $11.5 \%$ & $11.2 \%$ \\
\hline \multicolumn{16}{|l|}{ Health Insurer } \\
\hline Private & $69.2 \%$ & $65.5 \%$ & $64.3 \%$ & $63.3 \%$ & $63.4 \%$ & $62.2 \%$ & $61.2 \%$ & $59.7 \%$ & $59.5 \%$ & $59.1 \%$ & $59.6 \%$ & $60.2 \%$ & $63.6 \%$ & $62.6 \%$ & $63.9 \%$ \\
\hline Medicare & $2.9 \%$ & $2.7 \%$ & $2.8 \%$ & $3.0 \%$ & $3.1 \%$ & $3.0 \%$ & $3.3 \%$ & $3.3 \%$ & $3.3 \%$ & $4.0 \%$ & $4.0 \%$ & $3.9 \%$ & $5.1 \%$ & $4.9 \%$ & $4.9 \%$ \\
\hline Medicaid & $6.9 \%$ & $8.0 \%$ & $8.6 \%$ & $9.0 \%$ & $8.5 \%$ & $9.0 \%$ & $9.8 \%$ & $10.2 \%$ & $10.7 \%$ & $11.4 \%$ & $11.3 \%$ & $11.6 \%$ & $11.7 \%$ & $14.3 \%$ & $14.7 \%$ \\
\hline Tricare or VA & $1.4 \%$ & $1.7 \%$ & $1.6 \%$ & $1.7 \%$ & $1.6 \%$ & $1.6 \%$ & $1.7 \%$ & $1.8 \%$ & $2.0 \%$ & $2.1 \%$ & $2.1 \%$ & $2.1 \%$ & $2.1 \%$ & $2.0 \%$ & $2.2 \%$ \\
\hline Other & $1.8 \%$ & $2.4 \%$ & $2.4 \%$ & $2.4 \%$ & $2.5 \%$ & $2.8 \%$ & $2.6 \%$ & $2.8 \%$ & $2.9 \%$ & $2.8 \%$ & $3.0 \%$ & $2.9 \%$ & $3.0 \%$ & $3.7 \%$ & $3.0 \%$ \\
\hline Uninsured & $17.7 \%$ & $19.8 \%$ & $20.2 \%$ & $20.7 \%$ & $21.0 \%$ & $21.5 \%$ & $21.4 \%$ & $22.2 \%$ & $21.7 \%$ & $20.6 \%$ & $20.0 \%$ & $19.3 \%$ & $14.6 \%$ & $12.5 \%$ & $11.4 \%$ \\
\hline \multicolumn{16}{|c|}{ Past-Year Substance Abuse or Dependence } \\
\hline Alcohol & $12.8 \%$ & $12.4 \%$ & $12.6 \%$ & $12.6 \%$ & $12.3 \%$ & $12.1 \%$ & $12.2 \%$ & $12.0 \%$ & $11.4 \%$ & $10.7 \%$ & $10.5 \%$ & $9.9 \%$ & $8.4 \%$ & $7.9 \%$ & $7.7 \%$ \\
\hline Pain Reliever & $0.9 \%$ & $0.8 \%$ & $0.9 \%$ & $1.1 \%$ & $1.0 \%$ & $1.2 \%$ & $1.3 \%$ & $1.3 \%$ & $1.2 \%$ & $1.2 \%$ & $1.3 \%$ & $1.1 \%$ & $0.9 \%$ & $1.0 \%$ & $0.9 \%$ \\
\hline Cocaine & $1.0 \%$ & $1.0 \%$ & $1.1 \%$ & $1.1 \%$ & $1.0 \%$ & $1.0 \%$ & $0.9 \%$ & $0.7 \%$ & $0.5 \%$ & $0.5 \%$ & $0.5 \%$ & $0.5 \%$ & $0.4 \%$ & $0.4 \%$ & $0.4 \%$ \\
\hline Hallucinogen & $0.4 \%$ & $0.3 \%$ & $0.3 \%$ & $0.3 \%$ & $0.3 \%$ & $0.3 \%$ & $0.3 \%$ & $0.3 \%$ & $0.3 \%$ & $0.2 \%$ & $0.2 \%$ & $0.2 \%$ & $0.1 \%$ & $0.2 \%$ & $0.2 \%$ \\
\hline Heroin & $0.1 \%$ & $0.1 \%$ & $0.2 \%$ & $0.2 \%$ & $0.2 \%$ & $0.2 \%$ & $0.2 \%$ & $0.3 \%$ & $0.2 \%$ & $0.3 \%$ & $0.4 \%$ & $0.4 \%$ & $0.3 \%$ & $0.3 \%$ & $0.4 \%$ \\
\hline Inhalant & $0.1 \%$ & $0.1 \%$ & $0.1 \%$ & $0.1 \%$ & $0.1 \%$ & $0.1 \%$ & $0.1 \%$ & $0.1 \%$ & $0.1 \%$ & $0.1 \%$ & $\begin{array}{l}< \\
0.1 \%\end{array}$ & $\begin{array}{l}< \\
0.1 \%\end{array}$ & $\begin{array}{l}< \\
0.1 \%\end{array}$ & $\begin{array}{l}< \\
0.1 \%\end{array}$ & $\begin{array}{l}< \\
0.1 \%\end{array}$ \\
\hline Marijuana & $3.4 \%$ & $3.5 \%$ & $3.6 \%$ & $3.3 \%$ & $3.3 \%$ & $3.2 \%$ & $3.4 \%$ & $3.3 \%$ & $3.2 \%$ & $3.1 \%$ & $3.2 \%$ & $3.0 \%$ & $2.3 \%$ & $2.3 \%$ & $2.3 \%$ \\
\hline Sedative & $0.1 \%$ & $0.1 \%$ & $0.1 \%$ & $0.1 \%$ & $0.1 \%$ & $0.1 \%$ & $0.1 \%$ & $0.1 \%$ & $0.1 \%$ & $\begin{array}{l}< \\
0.1 \%\end{array}$ & $0.1 \%$ & $0.1 \%$ & $0.1 \%$ & $0.1 \%$ & $0.1 \%$ \\
\hline Stimulant & $0.3 \%$ & $0.3 \%$ & $0.3 \%$ & $0.3 \%$ & $0.2 \%$ & $0.3 \%$ & $0.2 \%$ & $0.3 \%$ & $0.2 \%$ & $0.2 \%$ & $0.3 \%$ & $0.3 \%$ & $0.3 \%$ & $0.3 \%$ & $0.3 \%$ \\
\hline Tranquilizer & $0.3 \%$ & $0.3 \%$ & $0.4 \%$ & $0.3 \%$ & $0.3 \%$ & $0.3 \%$ & $0.3 \%$ & $0.3 \%$ & $0.3 \%$ & $0.2 \%$ & $0.3 \%$ & $0.3 \%$ & $0.2 \%$ & $0.3 \%$ & $0.3 \%$ \\
\hline $\begin{array}{l}\text { Past-Year Unmet Need for Mental } \\
\text { Health Treatment }\end{array}$ & $7.4 \%$ & $7.1 \%$ & $7.2 \%$ & $7.2 \%$ & $6.4 \%$ & $6.8 \%$ & $6.8 \%$ & $7.1 \%$ & $6.6 \%$ & $6.6 \%$ & $6.7 \%$ & $6.6 \%$ & $6.4 \%$ & $6.3 \%$ & $6.8 \%$ \\
\hline
\end{tabular}

000-\$74,999 were more likely to indicate low perceived effectiveness of treatment as a reason for unmet need. In addition, respondents aged 50 and over, females, nonHispanic Asian respondents, and respondents with at least some high school education were more likely to cite structural barriers as a reason for unmet need.

Several groups had higher odds of reporting reasons other than those shown above as a cause for unmet need: respondents aged 26-49, non-Hispanic mixed respondents, those not working full-time as well as respondents insured by Tricare or VA or those reporting tranquilizer abuse Notably, those with at least some high school education showed much higher odds of reporting a reason not listed above as a cause of unmet need than those with an elementary school education.

\section{Discussion}

Our analyses have elucidated the major characteristics associated with increased odds of unmet need, which include: past year substance abuse or dependence (other than hallucinogens and sedatives), fair, poor, or very poor health, being female, and an educational attainment

Table 3 Reason(s) for unmet need among adults reporting unmet need for mental health treatment in the past year, $2002-16$

\begin{tabular}{|c|c|c|c|c|c|c|c|c|c|c|c|c|c|c|c|}
\hline Year & 2002 & 2003 & 2004 & 2005 & 2006 & 2007 & 2008 & 2009 & 2010 & 2011 & 2012 & 2013 & 2014 & 2015 & 2016 \\
\hline$n$ & 2680 & 2640 & 2665 & 2678 & 2368 & 2557 & 2530 & 2687 & 2556 & 2564 & 2537 & 2463 & 2652 & 2718 & 2891 \\
\hline \multicolumn{16}{|l|}{ Reason for Unmet Need } \\
\hline Cost & $61.8 \%$ & $62.3 \%$ & $63.8 \%$ & $63.0 \%$ & $47.3 \%$ & $47.3 \%$ & $48.7 \%$ & $50.8 \%$ & $50.9 \%$ & $51.6 \%$ & $48.4 \%$ & $48.5 \%$ & $48.5 \%$ & $44.5 \%$ & $43.8 \%$ \\
\hline Perceived Stigma & $31.2 \%$ & $32.1 \%$ & $30.8 \%$ & $30.2 \%$ & $29.7 \%$ & $31.8 \%$ & $31.1 \%$ & $30.5 \%$ & $32.1 \%$ & $29.5 \%$ & $31.0 \%$ & $30.2 \%$ & $30.4 \%$ & $32.8 \%$ & $34.9 \%$ \\
\hline Minimisation & $13.8 \%$ & $34.8 \%$ & $34.5 \%$ & $32.3 \%$ & $31.5 \%$ & $32.5 \%$ & $30.3 \%$ & $30.6 \%$ & $30.0 \%$ & $27.9 \%$ & $29.9 \%$ & $29.2 \%$ & $28.4 \%$ & $31.6 \%$ & $33.0 \%$ \\
\hline Low Perceived Effectiveness & $1.6 \%$ & $11.8 \%$ & $11.3 \%$ & $10.8 \%$ & $10.4 \%$ & $11.8 \%$ & $10.0 \%$ & $11.0 \%$ & $10.9 \%$ & $10.3 \%$ & $10.3 \%$ & $9.9 \%$ & $10.1 \%$ & $12.6 \%$ & $11.6 \%$ \\
\hline Structural Barriers & $28.8 \%$ & $34.8 \%$ & $32.9 \%$ & $31.7 \%$ & $29.9 \%$ & $31.9 \%$ & $32.5 \%$ & $31.9 \%$ & $32.1 \%$ & $32.2 \%$ & $33.6 \%$ & $35.8 \%$ & $35.8 \%$ & $41.8 \%$ & $42.2 \%$ \\
\hline Other Reason(s) & $4.4 \%$ & $8.3 \%$ & $7.4 \%$ & $7.9 \%$ & $7.3 \%$ & $7.8 \%$ & $7.4 \%$ & $7.6 \%$ & $7.3 \%$ & $7.7 \%$ & $7.2 \%$ & $7.9 \%$ & $8.3 \%$ & $9.2 \%$ & $9.7 \%$ \\
\hline
\end{tabular}


Table 4 Odds ratios and associated 95\% confidence intervals for unadjusted and adjusted multiple logistic regression of demographic, socioeconomic, and health characteristics associated with unmet need for mental health treatment for adults, 2002-16

\begin{tabular}{|c|c|c|c|c|}
\hline \multirow{2}{*}{$\overline{\text { Age }}$} & \multicolumn{2}{|c|}{ Unadjusted } & \multicolumn{2}{|c|}{ Adjusted } \\
\hline & & & & \\
\hline 18-25 years old & 1 & & 1 & \\
\hline 26-34 years old & 0.886 & $(0.853-0.920)$ & 1.056 & $(1.007-1.108)$ \\
\hline 35-49 years old & 0.698 & $(0.675-0.722)$ & 0.895 & $(0.851-0.941)$ \\
\hline 50 or older & 0.293 & $(0.277-0.311)$ & 0.364 & $(0.336-0.395)$ \\
\hline Female & 2.031 & $(1.952-2.113)$ & 2.299 & $(2.204-2.399)$ \\
\hline \multicolumn{5}{|l|}{ Ethnicity } \\
\hline Non-Hispanic White & 1 & & 1 & \\
\hline Non-Hispanic Black & 0.840 & $(0.789-0.895)$ & 0.568 & $(0.530-0.610)$ \\
\hline Non-Hispanic Native American or Alaskan Native & 1.315 & $(1.135-1.525)$ & 0.795 & $(0.676-0.934)$ \\
\hline Non-Hispanic Native Hawaiian or Pacific Islander & 0.640 & $(0.464-0.881)$ & 0.478 & $(0.339-0.672)$ \\
\hline Non-Hispanic Asian & 0.405 & $(0.351-0.467)$ & 0.376 & $(0.326-0.433)$ \\
\hline Non-Hispanic Mixed & 1.697 & $(1.497-1.925)$ & 1.244 & $(1.094-1.416)$ \\
\hline Hispanic & 0.734 & $(0.693-0.777)$ & 0.547 & $(0.510-0.586)$ \\
\hline \multicolumn{5}{|l|}{ Marital Status } \\
\hline Never married & 1 & & 1 & \\
\hline Married & 0.428 & $(0.413-0.445)$ & 0.755 & $(0.716-0.797)$ \\
\hline Widowed & 0.320 & $(0.282-0.364)$ & 0.612 & $(0.528-0.709)$ \\
\hline Divorced or Separated & 0.960 & $(0.914-1.009)$ & 1.228 & $(1.154-1.306)$ \\
\hline \multicolumn{5}{|l|}{ MSA/CBSA Size } \\
\hline 1 million or more & 1 & & 1 & \\
\hline Fewer than 1 million & 1.090 & $(1.056-1.125)$ & 0.983 & $(0.951-1.016)$ \\
\hline Not a MSA/CBSA & 0.946 & $(0.895-1.001)$ & 0.854 & $(0.801-0.911)$ \\
\hline \multicolumn{5}{|l|}{ Educational Attainment } \\
\hline Elementary School & 1 & & 1 & \\
\hline Middle School & 1.307 & $(0.972-1.758)$ & 1.093 & $(0.804-1.487)$ \\
\hline High School & 1.709 & $(1.322-2.211)$ & 1.301 & $(0.991-1.708)$ \\
\hline College or Higher & 2.062 & $(1.600-2.658)$ & 2.043 & $(1.563-2.671)$ \\
\hline \multicolumn{5}{|l|}{ Employment Status } \\
\hline Full Time & 1 & & 1 & \\
\hline Part Time & 1.552 & $(1.478-1.629)$ & 1.175 & $(1.115-1.239)$ \\
\hline Unemployed & 1.914 & $(1.784-2.052)$ & 1.226 & $(1.136-1.323)$ \\
\hline Other (including not in labour force) & 1.118 & $(1.069-1.169)$ & 1.128 & $(1.069-1.190)$ \\
\hline \multicolumn{5}{|l|}{ Annual Household Income } \\
\hline Less than $\$ 20,000$ & 1 & & 1 & \\
\hline$\$ 20,000-\$ 49,999$ & 0.687 & $(0.659-0.717)$ & 0.953 & $(0.907-1.000)$ \\
\hline$\$ 50,000-\$ 74,999$ & 0.580 & $(0.551-0.610)$ & 0.897 & $(0.842-0.955)$ \\
\hline$\$ 75,000$ or more & 0.475 & $(0.452-0.498)$ & 0.785 & $(0.740-0.833)$ \\
\hline Receives Government Assistance & 2.045 & $(1.969-2.123)$ & 1.461 & $(1.389-1.537)$ \\
\hline \multicolumn{5}{|l|}{ Self-Rated Health } \\
\hline Good, very good, or excellent & 1 & & 1 & \\
\hline Fair, poor, or very poor & 1.985 & $(1.905-2.069)$ & 2.433 & $(2.320-2.552)$ \\
\hline
\end{tabular}

Health Insurer 
Table 4 Odds ratios and associated 95\% confidence intervals for unadjusted and adjusted multiple logistic regression of demographic, socioeconomic, and health characteristics associated with unmet need for mental health treatment for adults, 2002-16 (Continued)

\begin{tabular}{|c|c|c|c|c|}
\hline & \multicolumn{2}{|c|}{ Unadjusted } & \multicolumn{2}{|c|}{ Adjusted } \\
\hline Private & 1 & & 1 & \\
\hline Medicare & 0.992 & $(0.910-1.082)$ & 1.074 & $(0.965-1.197)$ \\
\hline Medicaid & 2.452 & $(2.336-2.574)$ & 1.196 & $(1.118-1.280)$ \\
\hline Tricare or VA & 1.662 & $(1.480-1.867)$ & 1.573 & $(1.388-1.783)$ \\
\hline Other & 1.630 & $(1.462-1.816)$ & 1.086 & $(0.965-1.221)$ \\
\hline Uninsured & 1.831 & $(1.750-1.917)$ & 1.310 & $(1.241-1.382)$ \\
\hline \multicolumn{5}{|c|}{ Past-Year Substance Abuse or Dependence } \\
\hline Alcohol & 3.629 & $(3.471-3.795)$ & 2.628 & $(2.499-2.763)$ \\
\hline Pain Relievers & 8.443 & $(7.697-9.261)$ & 2.616 & $(2.304-2.970)$ \\
\hline Cocaine & 7.340 & $(6.651-8.101)$ & 2.056 & $(1.785-2.369)$ \\
\hline Hallucinogens & 8.146 & $(6.778-9.790)$ & 1.266 & $(0.957-1.675)$ \\
\hline Heroin & 7.656 & $(6.485-9.038)$ & 1.471 & $(1.112-1.946)$ \\
\hline Inhalants & 9.192 & $(6.234-13.55)$ & 2.325 & $(1.351-4.002)$ \\
\hline Marijuana & 4.678 & (4.383-4.993) & 1.955 & $(1.800-2.123)$ \\
\hline Sedatives & 16.73 & $(11.90-23.51)$ & 1.546 & $(0.832-2.873)$ \\
\hline Stimulants & 12.77 & $(10.60-15.37)$ & 2.156 & $(1.623-2.864)$ \\
\hline Tranquilizers & 12.35 & $(10.37-14.70)$ & 1.834 & $(1.392-2.418)$ \\
\hline
\end{tabular}

of college or higher. With respect to reasons for unmet need, cost was most often cited, followed by perceived stigma, structural barriers, and minimisation. Characteristics associated with increased odds of indicating cost as a reason for unmet need include: being uninsured or aged 26-35. Minimisation and low perceived effectiveness are mentioned by high-income persons as reasons for unmet need. College-educated persons and women had higher odds of citing structural barriers as a reason for unmet need.

Our study has some limitations such as using household survey data to assess unmet need as well as demographic, socioeconomic, and health characteristics of the sample population. Perceived unmet need is also a self-reported variable which was not validated using psychiatric diagnostic information; consequently, underreporting or over-reporting of perceived unmet need would affect the accuracy of prevalence estimates for unmet need. Indeed, perceived unmet need is subjective, based on sociocultural factors such as patient expectations and Allin and Masseria suggest that analyses of unmet need are contingent upon the specific phrasing of questions [22]. Small samples for specific subpopulations in this study limit the ability to identify specific patterns of unmet need in these populations. Moreover, the NSDUH excludes individuals with no fixed household address and those living in institutional premises, such as prisons, precluding conclusions on unmet need among these vulnerable populations. In addition, given the repeated, cross-sectional nature of the NSDUH, we are not able to conduct analyses of individuals over time and, therefore, we are unable to determine causality between unmet need and the demographic, socioeconomic, and health correlates under examination. Indeed, there are a number of other potential reasons for and contributors to unmet need which are not examined in this study which bear further scrutiny and study. Nevertheless, the NSDUH has been shown to provide comparable findings to other validated health studies such as the National Comorbidity Survey Replication (NCS-R) and is the only source of data in the United States which provides information on unmet need for a nationally representative sample of adults living in the United States [23]. Our results are consistent with recent and established literature which identify disparities in expressed unmet need based upon age [24], gender [25, 26], economic disadvantage [27], urban/rural status [28], health insurer [29], illicit substance use [30].

\section{Conclusion}

This study extends our understanding of disparities in mental health treatment by not only considering demographic, socioeconomic, and health characteristics of those expressing unmet need from 2002 to 16 but also identifying how these correlate with reasons for unmet need. Cost was a major cause of unmet need among respondents aged 26-49 and those who were uninsured; though the ACA has attempted to reduce the number of uninsured young adults, provisions only guarantee continued enrolment of dependent children until age 25 and, consequently, this may partly explain this observed pattern of expressed unmet need due to cost among these 


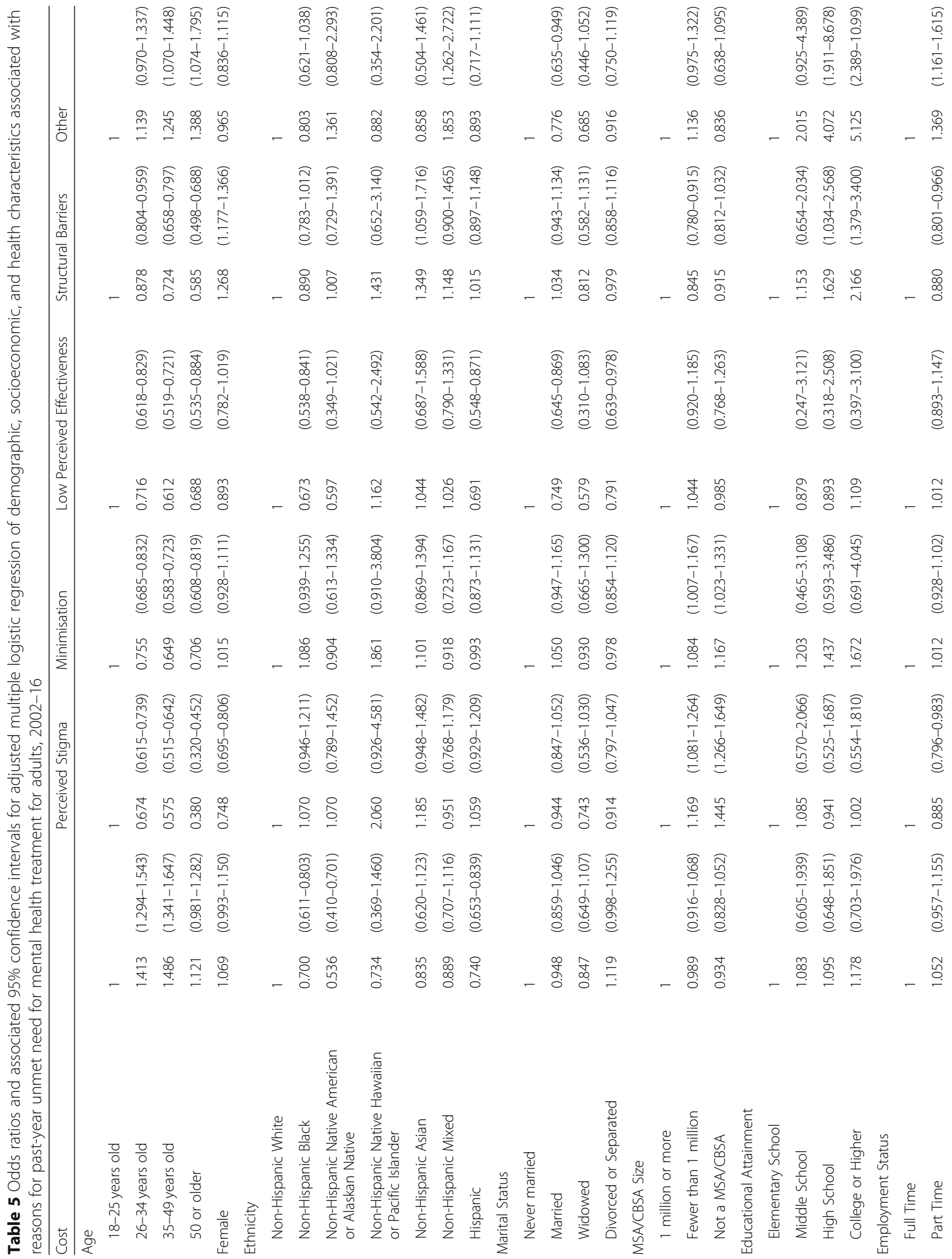




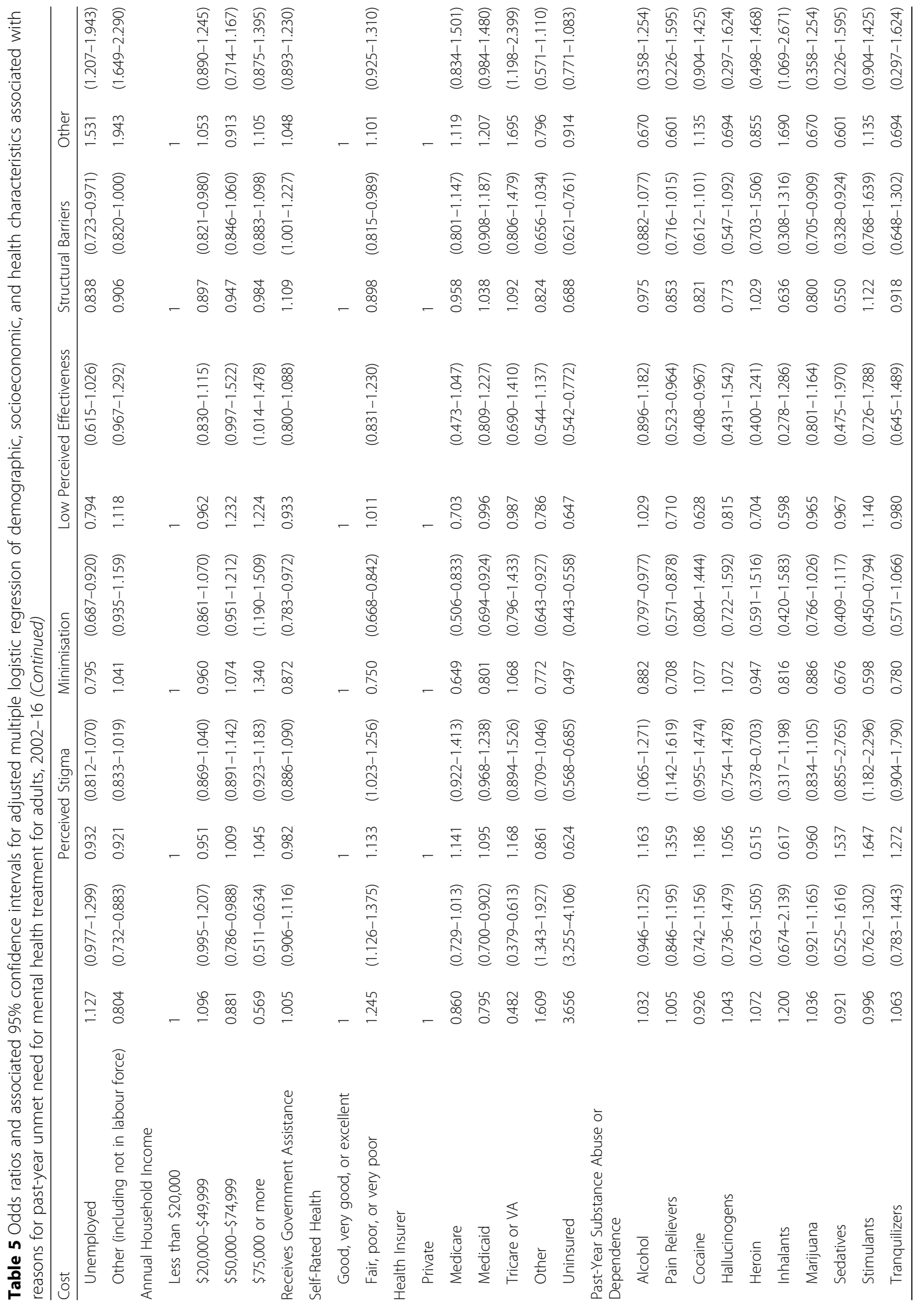


subgroups [31]. Of the reasons for unmet need examined in this study, only perceived stigma and minimisation appear to increase the odds of expressed unmet need among those living in suburban or rural areas, controlling for other demographic, socioeconomic, and health characteristics, highlighting a potential opportunity to develop health promotion interventions for these subpopulations to address unmet need consistent with established literature [32-35]. That the odds of indicating structural barriers as a reason for unmet need were not statistically significantly higher among those living in suburban or rural areas is somewhat surprising, given existing research which indicate that availability of adequate mental health care is a cause for concern [34, 36]. This, in turn, suggests that further research is needed to fully understand the availability or lack thereof of mental health treatment in suburban or rural areas which can inform new initiatives focused on access, such as telemedicine approaches [37, 38]. In addition, the large number of subpopulations expressing unmet need for reasons not specified in this study (i.e. cost, perceived stigma, minimisation, low perceived effectiveness of treatment, and structural barriers) suggests a need to investigate the reasons why these subpopulations express unmet need to address the causal factors underlying why these subpopulations do not receive adequate mental health care

\section{Acknowledgements}

Not applicable.

\section{Authors' contributions}

$J C Y$ and ARU conceived and designed the analysis. JCY analysed and interpreted the data. JCY and ARU drafted the manuscript. CB and MM provided critical revisions of the manuscript. All authors read and approved the final manuscript.

\section{Funding}

This study was not funded by any institution.

\section{Availability of data and materials}

The datasets supporting the conclusions of this article are available in the Substance Abuse \& Mental Health Data Archive repository, https://www. datafiles.samhsa.gov/study-series/national-survey-drug-use-and-health-nsduhnid13517.

\section{Ethics approval and consent to participate}

This article does not contain any studies with human participants or animals performed by any of the authors.

\section{Consent for publication}

Not applicable.

\section{Competing interests}

The authors declare that they have no competing interests.

\section{Author details}

'Department of Public Health \& Primary Care, Institute of Public Health, University of Cambridge, Forvie Site, Robinson Way, Cambridge CB2 OSR, UK. ${ }^{2}$ Department of Health Services Research and Policy, London School of Hygiene \& Tropical Medicine, London, UK.
Received: 22 March 2019 Accepted: 28 July 2019

Published online: 05 August 2019

\section{References}

1. GBD Compare. https://vizhub.healthdata.org/gbd-compare/. Accessed 16 Oct 2018 .

2. Walker ER, Druss BG. Cumulative burden of comorbid mental disorders, substance use disorders, chronic medical conditions, and poverty on health among adults in the U.S.A. Psychol Health Med. 2017;22:727-35.

3. Beronio K, Glied S, Frank R. How the affordable care act and mental health parity and addiction equity act greatly expand coverage of behavioral health care. J Behav Health Serv Res. 2014;41:410-28.

4. Creedon TB, Cook BL. Access to mental health care increased but not for substance use, while disparities remain. Health Aff (Millwood). 2016;35:1017-21.

5. Alang SM. Sociodemographic disparities associated with perceived causes of unmet need for mental health care. Psychiatr Rehabil J. 2015;38:293-9.

6. Priester MA, Browne T, lachini A, Clone S, DeHart D, Seay KD. Treatment access barriers and disparities among individuals with co-occurring mental health and substance use disorders: an integrative literature review. J Subst Abus Treat. 2016;61:47-59.

7. United States Department of H. Human services. Substance a, mental health services administration. Office of Applied S: National Survey on drug use and health, 2002. In: Inter-university consortium for political and social research [distributor]; 2015.

8. United States Department of H. Human Services. Substance a, mental health services administration. Office of Applied S: National Survey on drug use and health, 2003. In: Inter-university consortium for political and social research [distributor]; 2015.

9. United States Department of $\mathrm{H}$. Human services. Substance a, mental health services administration. Office of Applied S: National Survey on drug use and health, 2004. In: Inter-university consortium for political and social research [distributor]; 2015

10. United States Department of $\mathrm{H}$. Human services. Substance a, mental health services administration. Office of Applied S: National Survey on drug use and health, 2005. In: Inter-university consortium for political and social research [distributor]; 2015.

11. United States Department of $\mathrm{H}$. Human services. Substance a, mental health services administration. Office of Applied S: National Survey on drug use and health, 2006. In: Inter-university consortium for political and social research [distributor]; 2013.

12. United States Department of H. Human services. Substance a, mental health services administration. Office of Applied S: National Survey on drug use and health, 2007. In: Inter-university consortium for political and social research [distributor]; 2015.

13. United States Department of H. Human services. Substance a, mental health services administration. Office of Applied S: National Survey on drug use and health, 2008. In: Inter-university consortium for political and social research [distributor]; 2015.

14. United States Department of $\mathrm{H}$. Human services. Substance a, mental health services administration. Office of Applied S: National Survey on drug use and health, 2009. In: Inter-university consortium for political and social research [distributor]; 2015.

15. United States Department of $\mathrm{H}$. Human services. Substance a, mental health services administration. Center for Behavioral Health S, quality: National Survey on drug use and health, 2010. In: Inter-university consortium for political and social research [distributor]; 2015.

16. United States Department of $\mathrm{H}$. Human services. Substance a, mental health services administration. Center for Behavioral Health S, quality: National Survey on drug use and health, 2011. In: Inter-university consortium for political and social research [distributor]; 2015.

17. United States Department of H. Human services. Substance a, mental health services administration. Center for Behavioral Health S, quality: National Survey on drug use and health, 2012. In: Inter-university consortium for political and social research [distributor]; 2015.

18. United States Department of $\mathrm{H}$. Human services. Substance a, mental health services administration. Center for Behavioral Health S, quality: National Survey on drug use and health, 2013. In: Inter-university consortium for political and social research [distributor]; 2015.

19. United States Department of $\mathrm{H}$. Human services. Substance a mental health services administration. Center for Behavioral Health S, quality: National 
Survey on drug use and health, 2014. In: Inter-university consortium for political and social research [distributor]; 2016.

20. United States Department of H. Human services. Substance a, mental health services administration. Center for Behavioral Health S, quality: National Survey on drug use and health, 2015. In: RTI International; 2018.

21. United States Department of H. Human services. Substance a, mental health services administration. Center for Behavioral Health S, quality: National Survey on drug use and health, 2016. In: RTI International; 2018.

22. Allin S, Masseria C. Unmet need as an indicator of health care access. Eurohealth. 2009;15:7.

23. Merikangas KR, McClair VL. Epidemiology of substance use disorders. Hum Genet. 2012;131:779-89.

24. Choi NG, DiNitto DM, Marti CN. Alcohol and other substance use, mental health treatment use, and perceived unmet treatment need: comparison between baby boomers and older adults. Am J Addict. 2015;24:299-307.

25. Manuel JI, Stebbins MB, Wu E. Gender differences in perceived unmet treatment needs among persons with and without co-occurring disorders. J Behav Health Serv Res. 2018;45:1-12.

26. Chen LY, Strain EC, Crum RM, Mojtabai R. Gender differences in substance abuse treatment and barriers to care among persons with substance use disorders with and without comorbid major depression. J Addict Med. 2013;7:325-34.

27. Oh S, DiNitto DM, Kim Y. Substance use and use disorders and treatment receipt among adults in families receiving temporary assistance for needy families (TANF), 2003-2014. Addict Behav. 2018;85:173-9.

28. Chavez $\sqcup$, Kelleher KJ, Matson SC, Wickizer TM, Chisolm DJ. Mental health and substance use care among young adults before and after affordable care act (ACA) implementation: a rural and urban comparison. J Rural Health. 2018:34:42-7.

29. Wang $N$, Xie X. The impact of race, income, drug abuse and dependence on health insurance coverage among US adults. Eur J Health Econ. 2017;18:537-46.

30. Smith LL, Yan F, Charles M, Mohiuddin K, Tyus D, Adekeye O, Holden KB. Exploring the link between substance use and mental health status: what can we learn from the self-medication theory? J Health Care Poor Underserved. 2017;28:113-31.

31. Monaghan M. The affordable care act and implications for young adult health. Transl Behav Med. 2014;4:170-4.

32. Judd F, Jackson H, Komiti A, Murray G, Fraser C, Grieve A, Gomez RJA. Helpseeking by rural residents for mental health problems: the importance of agrarian values. Aust N Z J Psychiatry. 2006:40:769-76.

33. Fuller J, Edwards J, Procter N, Moss JJAJRH. How definition of mental health problems can influence help seeking in rural and remote communities. Aust J Rural Health. 2000:8:148-53.

34. Smalley KB, Yancey $C T$, Warren JC, Naufel $K$, Ryan $R$, JLJJocp P. Rural mental health and psychological treatment: a review for practitioners. J Clin Psychol. 2010;66:479-89.

35. Wrigley S, Jackson H, Judd F, Komiti AJA. Role of stigma and attitudes toward help-seeking from a general practitioner for mental health problems in a rural town. Aust N Z J Psychiatry. 2005;39:514-21.

36. Cherry S, Robinson A, Jashinsky J, Bagwell-Adams G, Elliott M, MJJoS D. Rural community health needs assessment findings: access to care and Mental Health. J Soc Behav Health Sci. 2017;11:18.

37. Wynn SD, Sherrod RA. Providing mental health care to veterans in rural areas: using telehealth in mobile clinics. J Psychosoc Nurs Ment Health Serv. 2012:50:22-8.

38. Barnwell SV, Juretic MA, Hoerster KD, Van de Plasch R, Felker BL. VA Puget Sound Telemental health service to rural veterans: a growing program. Psychol Serv. 2012;9:209-11.

\section{Publisher's Note}

Springer Nature remains neutral with regard to jurisdictional claims in published maps and institutional affiliations.

Ready to submit your research? Choose BMC and benefit from:

- fast, convenient online submission

- thorough peer review by experienced researchers in your field

- rapid publication on acceptance

- support for research data, including large and complex data types

- gold Open Access which fosters wider collaboration and increased citations

- maximum visibility for your research: over $100 \mathrm{M}$ website views per year

At BMC, research is always in progress.

Learn more biomedcentral.com/submissions 